\title{
ODD PERFECT NUMBERS, DIOPHANTINE EQUATIONS, AND UPPER BOUNDS
}

\author{
PACE P. NIELSEN
}

\begin{abstract}
We obtain a new upper bound for odd multiperfect numbers. If $N$ is an odd perfect number with $k$ distinct prime divisors and $P$ is its largest prime divisor, we find as a corollary that $10^{12} P^{2} N<2^{4^{k}}$. Using this new bound, and extensive computations, we derive the inequality $k \geq 10$.
\end{abstract}

\section{INTRODUCTION}

One of the oldest unsolved problems in mathematics is whether there exists an odd perfect number $N$. There are many roadblocks to the existence of such a number. For instance, from [14] we now know that $N>10^{1500}$ and $N$ has at least 101 prime factors (counting multiplicity). If $k$ is the number of distinct prime factors, then as proved in [12,13] we have $k \geq 9$ and $N<2^{4^{k}}$. A list of other restrictions can be found in 13 .

While work with odd perfect numbers has been mostly computational, the bound $N<2^{4^{k}}$ is a purely theoretical result. Due to its doubly exponential growth it has not been used seriously in calculations. In this paper we find a way to make this upper bound an effective estimation tool. As an application, we are able to prove that an odd perfect number must have at least 10 distinct prime factors.

The following is a brief outline of the paper. In 1 the focus is on generalizing the upper bound $N<2^{4^{k}}$, and improving it in a small, but important, way. This generalization works for odd multiperfect numbers, for the spoof odd perfect numbers, and in more abstract settings. In 92 , by applying this new upper bound we show that if $p$ and $q$ are distinct primes and $p^{a} q^{b} \| N$, then there are reasonably sized bounds on $a$ and $b$ in terms of $k$, the number of distinct prime divisors. Due to a massive computation, taking a few months, it follows that $\operatorname{gcd}\left(\sigma\left(p^{a}\right), \sigma\left(q^{b}\right)\right)$ has only moderately sized prime divisors. In $\$ 3$ we describe improvements to the methods in [13], taking advantage of the new information we have. This is followed by another computer calculation, proving that any odd perfect number has at least 10 distinct prime factors. Finally, in $\$$ we describe one of many roadblocks which prevents us from proving $k>10$.

Starting in \$2 readers should be familiar with basic facts about odd perfect numbers, including knowledge of congruence restrictions related to the special prime. As this paper is an extension of the methods used in [13, starting in 93 the reader should be familiar with the ideas in that paper.

Received by the editor June 14, 2013 and, in revised form, December 16, 2013.

2010 Mathematics Subject Classification. Primary 11N25; Secondary 11Y50.

Key words and phrases. Diophantine equation, perfect number. 


\section{A BetTer UPPER BOUND}

Let $N$ be a positive integer. Following the literature, $N$ is said (in increasing order of generality) to be perfect when $\sigma(N) / N=2$, multiperfect when $\sigma(N) / N \in$ $\mathbb{Z}$, and $n / d$-perfect when $\sigma(N) / N=n / d$. For simplicity, we will always assume $n, d \in \mathbb{Z}_{>0}$. Note that $n / d$ does not need to be in lowest terms. Writing $N=$ $\prod_{i=1}^{k} p_{i}^{e_{i}}$ where $p_{1}<\ldots<p_{k}$ are the prime divisors of $N$, the equation $\sigma(N) / N=$ $n / d$ can be rewritten as

$$
d \prod_{i=1}^{k}\left(\sum_{j=0}^{e_{i}} p_{i}^{j}\right)=n \prod_{i=1}^{k} p_{i}^{e_{i}} .
$$

This motivates us to look at the Diophantine equation

$$
d \prod_{i=1}^{k}\left(\sum_{j=0}^{e_{i}} x_{i}^{j}\right)=n \prod_{i=1}^{k} x_{i}^{e_{i}}
$$

in $k$ variables $x_{1}, \ldots, x_{k}$. It turns out that if we fix $k$ and look for integer solutions with the $x_{i}$ 's greater than 1 and odd, then there are finitely many solutions. In fact, there is an explicit upper bound on $\prod_{i=1}^{k} x_{i}^{e_{i}}$ in terms of $n, d$, and $k$, but independent of the $e_{i}$. Many of the ideas for this result flow from proofs in [4,7,12, As we will generalize and improve these results, and as some of the proofs are scattered in the literature, we include all the needed pieces here.

Lemma 1.1. Let $x_{1}, x_{2}, w \in \mathbb{R}_{>0}$, and assume $w<1$. We have

$$
\left(1-\frac{1}{x_{1}}\right)\left(1-\frac{1}{x_{2}}\right) \geq\left(1-\frac{1}{w x_{1}}\right)\left(1-\frac{1}{w^{-1} x_{2}}\right)
$$

if and only if $w \leq x_{2} / x_{1}$. Furthermore, equality holds in (2) if and only if $w=$ $x_{2} / x_{1}$. In particular, if $x_{1} \leq x_{2}$, then strict inequality holds in (2).

Proof. This follows from basic algebraic manipulation. Note that we can characterize equality and strict inequality in (2) under the much weaker assumption that $x_{1}, x_{2}$, and $w$ are nonzero. However, there is no need for this generality.

Lemma 1.2. Let $1<x_{1} \leq x_{2} \leq \ldots \leq x_{n}$ and $1<y_{1} \leq y_{2} \leq \ldots \leq y_{n}$ be nondecreasing sequences of real numbers satisfying

$$
\prod_{i=1}^{m} x_{i} \leq \prod_{i=1}^{m} y_{i}
$$

for every $m$ in the range $1 \leq m \leq n$. Then we have

$$
\prod_{i=1}^{n}\left(1-\frac{1}{x_{i}}\right) \leq \prod_{i=1}^{n}\left(1-\frac{1}{y_{i}}\right)
$$

where equality holds if and only if $x_{i}=y_{i}$ for every $i \geq 1$.

Proof. We follow Cook [4, but improve a little. We wish to minimize

$$
\prod_{i=1}^{n}\left(1-\frac{1}{y_{i}}\right)
$$

subject to the constraints that the $y_{i}$ form a nondecreasing sequence and satisfy (3) for each $m$. If we set $N=\prod_{i=1}^{n} x_{i}$, then lowering each $y_{i}$ which is bigger than 
$N$ down to $N$ will only decrease (5). Thus, we see that any minimizing solution belongs to the compact set inside the box $\left[x_{1}, N\right]^{n}$ subject to the constraints given in (3) and the constraint that the $y_{i}$ are nondecreasing. (The inequality $y_{1}>1$ is an open condition, but the closed condition $y_{1} \geq x_{1}$ implies it.) Thus we may fix the $y_{i}$ so that they in fact minimize (5).

Assume, by way of contradiction, that this minimizing solution does not agree with the $x_{i}$. We let $r$ be the first index where $x_{r} \neq y_{r}$, and so from (3) we have $x_{r}<y_{r}$. As the $y_{i}$ minimize (5), we see that $r<n$. We let $t$ be the largest index where $y_{t}=y_{r+1}$.

Define the new sequence

$$
z_{i}= \begin{cases}y_{i} & \text { if } i \neq r, t \\ w y_{r} & \text { if } i=r \\ w^{-1} y_{t} & \text { if } i=t\end{cases}
$$

for some real number $w$ with $0<w<1$ to be further specified shortly. Consider what happens if we replace the $y_{i}$ 's with the $z_{i}$ 's. First, we choose $w$ so that it satisfies $w>y_{r-1} / y_{r}$ (where $y_{r-1}=1$ in case $r=1$ ), and also $w>y_{t} / y_{t+1}$ (where this condition is vacuously satisfied if $t=n$ ). With these assumptions on $w$, the new sequence $\left\{z_{i}\right\}$ satisfies $1<z_{1} \leq z_{2} \leq \ldots \leq z_{n}$. Second, the quantity (5) decreases by Lemma 1.1. Third, (3) still holds when $m<r$ or $m \geq t$, since in those cases $\prod_{i=1}^{m} y_{i}=\prod_{i=1}^{m} z_{i}$.

We can make (3) hold for an $m$ in the interval $r \leq m<t$, if we have a strict inequality $\prod_{i=1}^{m} x_{i}<\prod_{i=1}^{m} y_{i}$ (by assuming $w>\prod_{i=1}^{m} x_{i} / y_{i}$ for each such $m$ ). Notice that this strict inequality does hold when $m=r$. However, if the strict inequality held for all $m$ in the interval $r<m<t$, then this would contradict our assumption that the $y_{i}$ 's were a minimizing choice.

Thus, we must have an equality

$$
\prod_{i=1}^{s} x_{i}=\prod_{i=1}^{s} y_{i}
$$

for some $s$ satisfying $r<s<t$. By the definition of $r$ we also have the equality $\prod_{i=1}^{r-1} x_{i}=\prod_{i=1}^{r-1} y_{i}$, hence

$$
\prod_{i=r}^{s} x_{i}=\prod_{i=r}^{s} y_{i} .
$$

Recall that $x_{r}<y_{r}$ and that $y_{r+1}=y_{r+2}=\cdots=y_{s}=\cdots=y_{t}$. Thus (7) turns into $\prod_{i=r+1}^{s} x_{i}>\prod_{i=r+1}^{s} y_{i}=y_{s}^{s-r}$. As the $x_{i}$ are a nondecreasing sequence, we have

$$
x_{s+1} \geq x_{s} \geq\left(\prod_{i=r+1}^{s} x_{i}\right)^{1 /(s-r)}>y_{s}=y_{s+1},
$$

where the last equality holds since $r+1 \leq s<t$ (using the definition of $t$ ). But then (6) implies $\prod_{i=1}^{s+1} x_{i}>\prod_{i=1}^{s+1} y_{i}$, contradicting (3) when $m=s+1$.

As we reached a contradiction in every case, this proves that the only minimizing solution is when $x_{i}=y_{i}$ for every $i \geq 1$.

Before putting the previous lemma to good use, we need one more straightforward result. 
Lemma 1.3. Let $r \in \mathbb{Z}_{>0}$ and define $F_{r}: \mathbb{R}_{\geq 1} \rightarrow \mathbb{R}_{\geq 0}$ by the rule $F_{r}(x)=$ $x^{2^{r}}-x^{2^{r-1}}$. The function $F_{r}$ is monotonically increasing.

Proof. Indeed, the derivative $F_{r}^{\prime}(x)=2^{r} x^{2^{r}-1}-2^{r-1} x^{2^{r-1}-1}=2^{r-1} x^{2^{r-1}-1}\left(2 x^{2^{r-1}}-1\right)$ is positive when $x \geq 1$.

The following is just a slight strengthening of [12, Lemma 1], allowing equality among the $x_{i}$ 's.

Lemma 1.4. Let $r, a, b \in \mathbb{Z}_{>0}$ and let $x_{1}, \ldots, x_{r}$ be integers with $1<x_{1} \leq \ldots \leq x_{r}$. If

$$
\prod_{i=1}^{r}\left(1-\frac{1}{x_{i}}\right) \leq \frac{a}{b}<\prod_{i=1}^{r-1}\left(1-\frac{1}{x_{i}}\right),
$$

then $a \prod_{i=1}^{r} x_{i} \leq(a+1)^{2^{r}}-(a+1)^{2^{r-1}}$.

Proof. Work by induction on $r \geq 1$. Notice that $a<b$ in any case. When $r=1$ we have $x_{1} \leq b /(b-a)$ which is maximized when $b=a+1$. Thus $a x_{1} \leq a(a+1)=$ $(a+1)^{2^{1}}-(a+1)^{2^{0}}$.

Now assume that $r \geq 2$ and also assume that the lemma holds for all integers smaller than $r$ (and for any choices for $a$ and $b$ ). Treating $a$ as a fixed constant, we can assume that $b$ has been chosen, along with integers $1<x_{1} \leq x_{2} \leq \ldots \leq x_{r}$, so that $\prod_{i=1}^{r} x_{i}$ is maximal and (8) holds. Next, set $n_{i}=(a+1)^{2^{i}-1}+1$ for $i<r$, and set $n_{r}=(a+1)^{2^{r-1}}$. We have $1<n_{1}<n_{2}<\ldots<n_{r}$ and

$$
\prod_{i=1}^{r}\left(1-\frac{1}{n_{i}}\right)=\frac{a}{a+1}<\prod_{i=1}^{r-1}\left(1-\frac{1}{n_{i}}\right) .
$$

Thus, from our maximality assumption,

$$
\prod_{i=1}^{r} n_{i} \leq \prod_{i=1}^{r} x_{i}
$$

If $a x_{1}<a n_{1}=(a+1)^{2}-1$, then after multiplying (8) by $\frac{x_{1}}{x_{1}-1}$ we have

$$
\prod_{i=2}^{r}\left(1-\frac{1}{x_{i}}\right) \leq \frac{a x_{1}}{b\left(x_{1}-1\right)}<\prod_{i=2}^{r-1}\left(1-\frac{1}{x_{i}}\right) .
$$

The induction hypothesis implies $\left(a x_{1}\right) \prod_{i=2}^{r} x_{i} \leq\left(a x_{1}+1\right)^{2^{r-1}}-\left(a x_{1}+1\right)^{2^{r-2}}<$ $(a+1)^{2^{r}}-(a+1)^{2^{r-1}}$. Thus we may as well assume $n_{1} \leq x_{1}$.

If $a x_{1} x_{2}<a n_{1} n_{2}=(a+1)^{4}-1$, then multiplying (8) by $\frac{x_{1} x_{2}}{\left(x_{1}-1\right)\left(x_{2}-1\right)}$ and performing a similar computation yields the upper bound we seek. Thus we may assume $n_{1} n_{2} \leq x_{1} x_{2}$. Repeating this argument, we have $\prod_{i=1}^{m} n_{i} \leq \prod_{i=1}^{m} x_{i}$ for $1 \leq m<r$. But this also holds when $m=r$ by (9).

Lemma 1.2 now applies, so we have

$$
\prod_{i=1}^{r}\left(1-\frac{1}{x_{i}}\right) \geq \prod_{i=1}^{r}\left(1-\frac{1}{n_{i}}\right)=\frac{a}{a+1} ;
$$


but as (8) holds for some $b$ we must have $b=a+1$. Again, appealing to Lemma 1.2. we have $x_{i}=n_{i}$ for all $i \geq 1$. In this case, we compute

$$
a \prod_{i=1}^{r} n_{i}=(a+1)^{2^{r}}-(a+1)^{2^{r-1}}
$$

as desired.

Remark. The bound given in the lemma is the best possible in case $b=a+1$. To simplify notation, throughout the paper we let $F_{r}$ be defined as in Lemma 1.3 . Thus, (8) says

$$
a \prod_{i=1}^{r} x_{i} \leq F_{r}(a+1)
$$

We also define $F_{0}(x):=x-1$.

Notation. Let $X$ be a finite set of integers. We write $\Pi(X)$ for $\prod_{x \in X} x$, with the empty product equaling 1 . We will also write $\Pi^{\prime}(X)$ for $\prod_{x \in X}(x-1)$.

The following lemma is an improvement on the author's work in [12, and also improves on the recent paper [3]. While seemingly innocuous, the improved result has far-reaching effects. (The main difference is the denominator of inequality (ii).)

Lemma 1.5 (cf. [12, Theorem 1], [7, Lemma 2]). Let $k, n, d \in \mathbb{Z}_{>0}$. Suppose (1) holds for some choice of positive integer exponents $\left\{e_{1}, \ldots, e_{k}\right\}$, and odd integers $X=\left\{x_{1}, \ldots, x_{k}\right\}$ each greater than 1 . Let $S$ be a (possibly empty) subset of $X$. There exist sets $S^{\prime}, S^{\prime \prime} \subseteq X$ satisfying $S \cap S^{\prime}=\emptyset, \emptyset \neq S^{\prime \prime} \subseteq S \cup S^{\prime}$, and such that if we let $w=\left|S^{\prime}\right|, v=\left|S^{\prime \prime}\right|, T=\left(S \cup S^{\prime}\right) \backslash S^{\prime \prime}, \delta=d \prod_{x_{i} \in S^{\prime \prime}} \sum_{j=0}^{e_{i}} x_{i}^{j}$, and $\nu=n \prod_{x_{i} \in S^{\prime \prime}} x_{i}^{e_{i}}$, then:

(i) $\delta \prod_{x_{i} \in X \backslash S^{\prime \prime}}\left(\sum_{j=0}^{e_{i}} x_{i}^{j}\right)=\nu \prod_{x_{i} \in X \backslash S^{\prime \prime}} x_{i}^{e_{i}}$.

(ii) $\delta \Pi(T)<\frac{1}{\Pi\left(S^{\prime \prime}\right) \Pi^{\prime}\left(S^{\prime \prime}\right)} F_{v+w}(d \Pi(S)+1)$.

Proof. We are assuming that the elements in $X$ are odd, and so the fraction $n / d$, when written in lowest terms, has odd denominator. In particular,

$$
\prod_{x_{i} \in S}\left(1-\frac{1}{x_{i}}\right) \neq \frac{d}{n} \text {. }
$$

Thus, we have two cases to consider.

Case 1. $\prod_{x_{i} \in S}\left(1-\frac{1}{x_{i}}\right)>\frac{d}{n}$.

In this case, set $d^{\prime}=d \Pi(S)$ and $n^{\prime}=n \Pi^{\prime}(S)$. From $\prod_{x_{i} \in S}\left(1-\frac{1}{x_{i}}\right)>\frac{d}{n}$ we see $d^{\prime} / n^{\prime}<1$. Further, we calculate

$$
\prod_{x_{i} \in X}\left(1-\frac{1}{x_{i}}\right)<\prod_{x_{i} \in X}\left(\frac{x_{i}-1}{x_{i}-\frac{1}{x_{i}^{e_{i}}}}\right)=\prod_{x_{i} \in X} \frac{x_{i}^{e_{i}}}{\sum_{j=0}^{e_{i}} x_{i}^{j}}=\frac{d}{n} .
$$

Therefore

$$
\prod_{x_{i} \notin S}\left(1-\frac{1}{x_{i}}\right)<\frac{d^{\prime}}{n^{\prime}}<1
$$


This implies that there is a subset $S^{\prime} \subseteq X \backslash S$ such that if we write $S^{\prime}=\left\{y_{1}, \ldots, y_{w}\right\}$ with $y_{1} \leq \ldots \leq y_{w}$, then

$$
\prod_{i=1}^{w}\left(1-\frac{1}{y_{i}}\right) \leq \frac{d^{\prime}}{n^{\prime}}<\prod_{i=1}^{w-1}\left(1-\frac{1}{y_{i}}\right)
$$

and so by Lemma 1.4 we have $d^{\prime} \Pi\left(S^{\prime}\right) \leq F_{w}\left(d^{\prime}+1\right)$. Using the definition of $d^{\prime}$ we rewrite this as

$$
d \Pi(S) \Pi\left(S^{\prime}\right) \leq F_{w}(d \Pi(S)+1) .
$$

Notice that we also have

$$
\prod_{x_{i} \in S \cup S^{\prime}}\left(1-\frac{1}{x_{i}}\right)<\frac{d}{n}
$$

This completes the construction of $S^{\prime}$ in Case 1.

Case 2. $\prod_{x_{i} \in S}\left(1-\frac{1}{x_{i}}\right)<\frac{d}{n}$.

We set $S^{\prime}=\emptyset($ so $w=0)$. We note that inequalities (12) and (13) still hold in this case.

The construction of the set $S^{\prime \prime}$ is the same in both Case 1 and Case 2. We only need to know that inequalities (12) and (13) hold in both cases, so we continue with the general construction. Put $d^{\prime \prime}=d \Pi(S) \Pi\left(S^{\prime}\right)$ and $n^{\prime \prime}=n \Pi^{\prime}(S) \Pi^{\prime}\left(S^{\prime}\right)$. Inequality (13) is equivalent to $n^{\prime \prime} / d^{\prime \prime}<1$, which we will use shortly. We calculate

$$
\prod_{x_{i} \in S \cup S^{\prime}} \frac{1-\frac{1}{x_{i}^{e_{i}+1}}}{1-\frac{1}{x_{i}}}=\prod_{x_{i} \in S \cup S^{\prime}} \frac{\sum_{j=0}^{e_{i}} x_{i}^{j}}{x_{i}^{e_{i}}} \leq \prod_{x_{i} \in X} \frac{\sum_{j=0}^{e_{i}} x_{i}^{j}}{x_{i}^{e_{i}}}=\frac{n}{d}
$$

and hence

$$
\prod_{x_{i} \in S \cup S^{\prime}}\left(1-\frac{1}{x_{i}^{e_{i}+1}}\right) \leq \frac{n^{\prime \prime}}{d^{\prime \prime}}<1 .
$$

We pick a subset $S^{\prime \prime}=\left\{z_{1}, \ldots, z_{v}\right\} \subseteq S \cup S^{\prime}$ such that $z_{1}^{e\left(z_{1}\right)+1} \leq \ldots \leq z_{v}^{e\left(z_{v}\right)+1}$ and

$$
\prod_{i=1}^{v}\left(1-\frac{1}{z_{i}^{e\left(z_{i}\right)+1}}\right) \leq \frac{n^{\prime \prime}}{d^{\prime \prime}}<\prod_{i=1}^{v-1}\left(1-\frac{1}{z_{i}^{e\left(z_{i}\right)+1}}\right) .
$$

(By $e\left(z_{i}\right)$ we mean the exponent corresponding to $z_{i}$.) By Lemma 1.4, and the inequality $n^{\prime \prime}<d^{\prime \prime}$, we have

$$
n^{\prime \prime} \prod_{x_{i} \in S^{\prime \prime}} x_{i}^{e_{i}+1} \leq F_{v}\left(n^{\prime \prime}+1\right) \leq F_{v}\left(d^{\prime \prime}\right)=F_{v}\left(d \Pi(S) \Pi\left(S^{\prime}\right)\right) .
$$

This completes the construction of $S^{\prime \prime}$. We now only need to verify properties (i) and (ii). Property (i) is obvious, coming from equation (11). For property (ii), we 
compute

$$
\begin{aligned}
& \delta \Pi(T)=d \prod_{x_{i} \in S^{\prime \prime}}\left(\frac{x_{i}^{e_{i}+1}-1}{x_{i}-1}\right) \frac{\Pi(S) \Pi\left(S^{\prime}\right)}{\Pi\left(S^{\prime \prime}\right)} \quad \text { by the definition of } \delta \text { and } T \\
& =\frac{1}{\Pi\left(S^{\prime \prime}\right) \Pi^{\prime}\left(S^{\prime \prime}\right)} d^{\prime \prime} \prod_{x_{i} \in S^{\prime \prime}}\left(x_{i}^{e_{i}+1}-1\right) \quad \text { by the definition of } d^{\prime \prime} \\
& \leq \frac{1}{\Pi\left(S^{\prime \prime}\right) \Pi^{\prime}\left(S^{\prime \prime}\right)} n^{\prime \prime} \prod_{x_{i} \in S^{\prime \prime}} x_{i}^{e_{i}+1} \quad \text { by inequality (14) } \\
& \leq \frac{1}{\Pi\left(S^{\prime \prime}\right) \Pi^{\prime}\left(S^{\prime \prime}\right)} F_{v}\left(d \Pi(S) \Pi\left(S^{\prime}\right)\right) \quad \text { by inequality (15) } \\
& \leq \frac{1}{\Pi\left(S^{\prime \prime}\right) \Pi^{\prime}\left(S^{\prime \prime}\right)} F_{v}\left(F_{w}(d \Pi(S)+1)\right) \quad \text { by inequality (12) } \\
& <\frac{1}{\Pi\left(S^{\prime \prime}\right) \Pi^{\prime}\left(S^{\prime \prime}\right)} F_{v+w}(d \Pi(S)+1) .
\end{aligned}
$$

Thus, we have established the needed inequality.

We are now ready to put all of these lemmas together to prove an improved upper bound on odd multiperfect numbers. The improvement from previous results is in the denominator term.

Theorem 1.6. Let $k, n, d \in \mathbb{Z}_{>0}$, and suppose equation (11) holds for some choice of positive integer exponents $\left\{e_{1}, \ldots, e_{k}\right\}$ and odd integers $X=\left\{x_{1}, \ldots, x_{k}\right\}$ each greater than 1 . In this case

$$
\prod_{i=1}^{k} x_{i}^{e_{i}}<\frac{F_{2 k}(d+1)}{n \Pi(X) \Pi^{\prime}(X)}<\frac{(d+1)^{2^{2 k}}}{n \Pi(X) \Pi^{\prime}(X)} .
$$

Proof. Let $X_{0}=X, n_{0}=n, d_{0}=d$, and $S_{0}=\emptyset$. Using Lemma 1.5, we can construct $S_{0}^{\prime}, S_{0}^{\prime \prime}, w_{0}, v_{0}, \nu_{0}, \delta_{0}$, and $T_{0}$ (using the same notation, just with the extra subscript) satisfying properties (i) and (ii). Thus

$$
\delta_{0} \prod_{x_{i} \in X_{0} \backslash S_{0}^{\prime \prime}}\left(\sum_{j=0}^{e_{i}} x_{i}^{j}\right)=\nu_{0} \prod_{x_{i} \in X_{0} \backslash S_{0}^{\prime \prime}} x_{i}^{e_{i}} .
$$

Putting $X_{1}=X_{0} \backslash S_{0}^{\prime \prime}, S_{1}=T_{0}, n_{1}=\nu_{0}$, and $d_{1}=\delta_{0}$, we see from equation (16) that we can again use Lemma 1.5. Hence, we can construct $S_{1}^{\prime}, S_{1}^{\prime \prime}, T_{1}^{\prime}$, and so forth. We continue this process of repeatedly using Lemma 1.5, increasing the indices at every step. Since $S_{i}^{\prime \prime} \neq \emptyset$, we see that $X_{0} \supsetneq X_{1} \supsetneq \ldots$, and so this process must terminate (in at least $k$ steps), say $X_{r+1}=\emptyset$. Further, we see that $\sum_{i=0}^{r} w_{i}=\sum_{i=0}^{r} v_{i}=k$ (since for each element $x \in X$ there are unique indices $i \leq j$ such that $x$ is added into $S_{i}^{\prime}$, and then put into $\left.S_{j}^{\prime \prime}\right)$.

Using property (ii), repeatedly, we have

$$
\begin{aligned}
& d_{r+1} \Pi\left(S_{r+1}\right)<\frac{1}{\Pi\left(S_{r}^{\prime \prime}\right) \Pi^{\prime}\left(S_{r}^{\prime \prime}\right)} F_{w_{r}+v_{r}}\left(d_{r} \Pi\left(S_{r}\right)+1\right) \\
& \quad \leq \frac{1}{\Pi\left(S_{r}^{\prime \prime}\right) \Pi^{\prime}\left(S_{r}^{\prime \prime}\right)} F_{w_{r}+v_{r}}\left(\frac{1}{\Pi\left(S_{r-1}^{\prime \prime}\right) \Pi^{\prime}\left(S_{r-1}^{\prime \prime}\right)} F_{w_{r-1}+v_{r-1}}\left(d_{r-1} \Pi\left(S_{r-1}\right)+1\right)+1\right) \\
& \quad<\frac{1}{\Pi\left(S_{r}^{\prime \prime}\right) \Pi^{\prime}\left(S_{r}^{\prime \prime}\right)} F_{w_{r}+v_{r}}\left(\frac{1}{\Pi\left(S_{r-1}^{\prime \prime}\right) \Pi^{\prime}\left(S_{r-1}^{\prime \prime}\right)}\left(d_{r-1} \Pi\left(S_{r-1}\right)+1\right)^{2^{w_{r-1}+v_{r-1}}}+1\right) .
\end{aligned}
$$


Note that $w_{r} \geq 1$, and $v_{i} \geq 1$ for each $i$. Also observe that $\left(d_{r-1} \Pi\left(S_{r-1}\right)+1\right)^{2^{w_{r-1}}} \geq$ $\Pi\left(S_{r-1}^{\prime \prime}\right)$ by (12). Thus

$$
\begin{gathered}
F_{w_{r}+v_{r}}\left(\frac{1}{\Pi\left(S_{r-1}^{\prime \prime}\right) \Pi^{\prime}\left(S_{r-1}^{\prime \prime}\right)}\left(d_{r-1} \Pi\left(S_{r-1}\right)+1\right)^{2^{w_{r-1}+v_{r-1}}}+1\right) \\
\leq F_{w_{r}+v_{r}}\left(\frac{1}{\Pi\left(S_{r-1}^{\prime \prime}\right)}\left(d_{r-1} \Pi\left(S_{r-1}\right)+1\right)^{2^{w_{r-1}+v_{r-1}}}\right) \\
\leq \frac{1}{\Pi\left(S_{r-1}^{\prime \prime}\right)^{2}} F_{w_{r}+v_{r}}\left(\left(d_{r-1} \Pi\left(S_{r-1}\right)+1\right)^{2^{w_{r-1}+v_{r-1}}}\right) \\
=\frac{1}{\Pi\left(S_{r-1}^{\prime \prime}\right)^{2}} F_{w_{r}+v_{r}+w_{r-1}+v_{r-1}}\left(d_{r-1} \Pi\left(S_{r-1}\right)+1\right) .
\end{gathered}
$$

Continuing our inequality from before, and repeating the ideas used in the computations above, we have

$$
\begin{aligned}
d_{r+1} \Pi\left(S_{r+1}\right) & <\frac{1}{\Pi\left(S_{r}^{\prime \prime}\right) \Pi^{\prime}\left(S_{r}^{\prime \prime}\right) \Pi\left(S_{r-1}^{\prime \prime}\right)^{2}} F_{w_{r-1}+w_{r}+v_{r-1}+v_{r}}\left(d_{r-1} \Pi\left(S_{r-1}\right)+1\right) \\
& <\frac{1}{\Pi\left(S_{r}^{\prime \prime} \cup S_{r-1}^{\prime \prime}\right) \Pi^{\prime}\left(S_{r}^{\prime \prime} \cup S_{r-1}^{\prime \prime}\right)} F_{w_{r-1}+w_{r}+v_{r-1}+v_{r}}\left(d_{r-1} \Pi\left(S_{r-1}\right)+1\right) \\
& <\ldots<\frac{1}{\Pi\left(\bigcup_{i=0}^{r} S_{i}^{\prime \prime}\right) \Pi^{\prime}\left(\bigcup_{i=0}^{r} S_{i}^{\prime \prime}\right)} F_{\sum_{i=0}^{r}\left(w_{i}+v_{i}\right)}\left(d_{0} \Pi\left(S_{0}\right)+1\right) \\
& =\frac{1}{\Pi(X) \Pi^{\prime}(X)} F_{2 k}\left(d_{0} \Pi\left(S_{0}\right)+1\right) .
\end{aligned}
$$

Now, $d_{0}=d, S_{0}=S_{r+1}=\emptyset$, and $d_{r+1}=d \prod_{x_{i} \in X} \frac{x_{i}^{e_{i}+1}-1}{x_{i}-1}=n \prod_{x_{i} \in X} x_{i}^{e_{i}}$. Plugging these values into the inequality above, we obtain the theorem.

Remark. It is believed that there are an infinite number of even perfect numbers. Such numbers necessarily have exactly two distinct prime factors. Thus, the previous theorem should (at least conjecturally) prove false if we do not stipulate that the $x_{i}$ are odd, even if we force the $x_{i}$ to be prime. In any case, we do have the infinite family of solutions to (1) when $k=2, x_{1}=2, x_{2}=2^{m}-1$, for $n=2$, $d=1, e_{1}=m-1$, and $e_{2}=1$, when we remove the hypothesis that the $x_{i}$ are odd. There also exist more exotic infinite families like $x_{1}=3, x_{2}=3, x_{3}=3^{m}-1$, for $n=2, d=1, e_{1}=1, e_{2}=m-1$, and $e_{3}=1$.

Remark. The hypotheses in the previous theorem are weak enough to capture the so-called "spoof" odd perfect number constructed by Descartes; $N=3^{2} 7^{2} 11^{2} 13^{2}$ $22021^{1}$, where $22021=19^{2} 61$ is treated as a prime. According to the work of Dittmer [5], there are no other spoofs of this sort with $k \leq 7$. On the other hand, our conditions are not weak enough to capture spoofs involving negative integers, such as $N=2^{3} 3^{2}(-5)^{1}(-13)^{1}$ which is attributed to Greg Martin.

Using a clever idea from [3] (which was independently suggested to me by Mitsuo Kobayashi), we can find a cleaner upper bound.

Corollary 1.7. Using the assumptions and notations of Theorem 1.6, and setting $N=\prod_{i=1}^{k} x_{i}^{e_{i}}$, the following chain of inequalities holds:

$$
N<d \frac{F_{2 k}(d+1)}{F_{k+1}(d+1)}<d(d+1)^{\left(2^{k}-1\right)^{2}} .
$$


In particular, when $N$ is an odd multiperfect number we achieve

$$
N<2^{\left(2^{k}-1\right)^{2}} .
$$

Proof. By hypothesis, we have the equality

$$
\prod_{i=1}^{k} \frac{x_{i}^{e_{i}+1}-1}{x_{i}^{e_{i}}\left(x_{i}-1\right)}=\frac{n}{d}
$$

which we can rewrite in the form

$$
\prod_{i=1}^{k}\left(1-\frac{1}{x_{i}^{e_{i}+1}}\right)=\frac{n \Pi^{\prime}(X)}{d \Pi(X)} .
$$

Applying Lemma 1.4 with $a=n \Pi^{\prime}(X)$ and $b=d \Pi(X)$, we see that

$$
n \Pi^{\prime}(X) \prod_{i=1}^{k} x_{i}^{e_{i}+1}=n \Pi^{\prime}(X) \Pi(X) N \leq F_{k}\left(n \Pi^{\prime}(X)+1\right) .
$$

After dividing both sides by $n \Pi^{\prime}(X) \Pi(X)$, and using the fact that $d \Pi(X) \geq$ $n \Pi^{\prime}(X)+1$, we arrive at the inequality

$$
N \leq d \frac{\left(n \Pi^{\prime}(X)+1\right)^{2^{k}}-\left(n \Pi^{\prime}(X)+1\right)^{2^{k-1}}}{\left(n \Pi^{\prime}(X)+1\right)^{2}-\left(n \Pi^{\prime}(X)+1\right)} .
$$

If $n \Pi^{\prime}(X)+1<(d+1)^{2^{k}}$, then as the quantity on the right-hand side of (19) is a strictly increasing function in terms of $n \Pi^{\prime}(X)+1$, we obtain the first inequality in (17). On the other hand, if $n \Pi^{\prime}(X)+1 \geq(d+1)^{2^{k}}$, then using the bound found in Theorem 1.6. we again achieve the first inequality in (17).

Now, we prove the second inequality in (17). As $\left(y^{a b}-1\right) /\left(y^{a}-1\right)=y^{a b-a}+$ $y^{a b-2 a}+\cdots+y^{a}+1<y^{a b-a+1}$ (for $y \geq 2$ ), we calculate

$$
\begin{aligned}
d \frac{(d+1)^{2^{2 k}}-(d+1)^{2^{2 k-1}}}{(d+1)^{2^{k+1}}-(d+1)^{2^{k}}} & =d(d+1)^{2^{2 k-1}-2^{k}} \frac{(d+1)^{2^{2 k-1}}-1}{(d+1)^{2^{k}}-1} \\
& <d(d+1)^{2^{2 k-1}-2^{k}+2^{2 k-1}-2^{k}+1}=d(d+1)^{\left(2^{k}-1\right)^{2}} .
\end{aligned}
$$

For the last statement, take $d=1$.

Those familiar with the bounds in 3, 12, will notice that these new bounds are not significantly smaller. For the purposes of this paper, we want to concentrate on the situation where $N$ is divisible by a large prime. Hence, in what follows we do not want to incorporate the $\Pi(X)$ and $\Pi^{\prime}(X)$ terms into an upper bound for $N$. This naturally leads to an inequality that seems much weaker, but will often suffice for our purposes.

Corollary 1.8. Let $N$ be an odd perfect number with $k$ distinct prime factors. If $P$ is the largest prime factor of $N$, then $10^{12} P^{2} N<2^{4^{k}}$.

Proof. Since $N$ is perfect we take $n=2$ and $d=1$. Clearly, $2(P-1)>P$. It is known, due to work of Iannucci [8, 9] that the second largest prime factor of $N$ is bigger than $10^{4}$, and the third largest prime factor is bigger than $10^{2}$. The corollary now follows by specializing the main result of Theorem [1.6 to this case. 
In the past, these types of theoretical upper bounds on odd perfect numbers were of little use in calculations due to their doubly exponential growth. However, in the next section we find a way to exploit the existence of very large prime divisors of $N$ (when they occur), which then makes the upper bound a feasible computational tool.

\section{Using THE GCD ALGORITHM}

In 13 it was proved that if $N$ is an odd perfect number with $k$ distinct prime factors, $p$ is a Fermat prime, and $p^{a} \| N$ with $a$ large, then the special prime factor of $N$ is large. In particular, we can use this fact in conjunction with Corollary 1.8 to obtain an upper bound on the size of the special prime, and hence on the size of $a$. To do so, we first need to recall a few well-known results.

The next three lemmas state some limitations on the exponent $a$. In particular, it cannot have too many prime divisors or be too large.

Lemma 2.1. Let $p$ be a prime and let $N$ be an odd perfect number with $k$ distinct prime divisors. If $p^{a} \| N$ with $a \in \mathbb{Z}_{>0}$, then $\sigma_{0}(a+1) \leq k$.

Proof. Weaken [13, Lemma 4].

Lemma 2.2. Let $p$ be a Fermat prime, let $N$ be an odd perfect number, and let $\pi$ be the special prime factor. Let $a, m \in \mathbb{Z}_{>0}$. If $p^{m} \mid(\pi+1)$ and $p^{a}|| N$ with $a<3 m$, then $\pi \nmid \sigma\left(p^{a}\right)$.

Proof. This is a specialization of [1, Lemma 1].

Lemma 2.3. Let $q$ be a Fermat prime, let $N$ be an odd perfect number with $k$ distinct prime factors, let $\pi$ be the special prime factor, and suppose $q^{a} \mid N$ for some $a \in \mathbb{Z}_{>0}$. If $a>(k-1)(k-2)$, then $2 q^{a-(k-2)(k-3)-\lfloor(k-2) / 2\rfloor} \mid(\pi+1)$.

Proof. This is a significant weakening of [13, Proposition 7]. (In the notation of that proposition, we take $b=0$ and assume $q$ is the only known prime. Thus $k_{2}=k-1$ and $k_{1}^{\prime}=\ell_{1}^{\prime}=0$.) Compare with [16, Proposition 3.1].

The following proposition takes these lemmas and in conjunction with the main theorem of the previous section finds strong upper bounds on the exponent $a$.

Proposition 2.4. Let $p$ and $q$ be odd, distinct primes less than 180 , and let $T=$ $\{3,5,17\}$. Let $N$ be an odd perfect number with $k \leq 9$ distinct prime divisors. Suppose $p^{a}\left\|N, q^{b}\right\| N$, and $10^{100}<p^{a}<q^{b}$ for some $a, b \in \mathbb{Z}_{>0}$.

(i) If $p, q \in T$, then

$$
a<\frac{1}{10}\left(\frac{4^{9} \log (2)+147 \log (q)-12 \log (10)}{\log (p)}+147\right) .
$$

(ii) If $p \in T$ and $q \notin T$, then

$$
a<\frac{1}{6}\left(\frac{4^{9} \log (2)+12 \log (q)-12 \log (10)}{\log (p)}+102\right) .
$$

(iii) If $p \notin T$ and $q \in T$, then

$$
a<\frac{1}{6}\left(\frac{4^{9} \log (2)+102 \log (q)-12 \log (10)}{\log (p)}+12\right) .
$$


(iv) If $p, q \notin T$, then

$$
a<\frac{1}{4}\left(\frac{4^{9} \log (2)+12 \log (q)-12 \log (10)}{\log (p)}+12\right) .
$$

Proof. A quick computer search, restricting to odd primes $p$ and $q$ less than 180 , demonstrates that if $q^{p-1} \equiv 1\left(\bmod p^{n}\right)$, then $n \leq 3$ except for the pair $(p, q)=$ $(3,163)$. For those pairs $(p, q) \neq(3,163)$, by Lemma 2.1] and [13, Lemma 2] we have $v_{p}\left(\sigma\left(q^{b}\right)\right) \leq k+3$. This inequality also holds for the exceptional pair $(p, q)=(3,163)$ since the multiplicative order of 163 modulo 3 is 1 and 163 cannot be the special prime. Similarly, $v_{q}\left(\sigma\left(p^{a}\right)\right) \leq k+3$. (We also note that there is only one pair with $q^{p-1} \equiv 1\left(\bmod p^{3}\right)$ for which similar reasoning does not allow us to use the slightly better bound $k+2$, namely, $(p, q)=(3,53)$. But such an improvement is not significant, and we will not pursue it here.)

By Corollary 1.8 we find

$$
2^{4^{9}}>10^{12} 2 N \geqslant 10^{12} 2 p^{a} q^{b}\left(\frac{\sigma\left(p^{a} q^{b}\right)}{2 p^{k+3} q^{k+3}}\right)>10^{12} \frac{p^{4 a-12}}{q^{12}} .
$$

Note that the term inside the large parentheses consists of divisors of $N$ relatively prime to $p$ and $q$ (possibly with extra factors in the denominator), which is how we obtain the second inequality. Solving for $a$ yields the bound in part (iv).

Now suppose for a moment that $p$ is a Fermat prime in $T$. Since $k \leq 9$, we have $(k-1)(k-2) \leq 56$. But $17^{80}<10^{100}$ so $a>80$. Lemma 2.3 then yields

$$
2 p^{a-45}\left|2 p^{a-(k-2)(k-3)-\lfloor(k-2) / 2\rfloor}\right|(\pi+1) .
$$

From $a>80$ we obtain $3(a-45)>a$, so by Lemma 2.2 we conclude that $\pi \nmid \sigma\left(p^{a}\right)$. If $q$ is a Fermat prime in $T$, then by the same analysis we obtain $\pi \nmid \sigma\left(q^{b}\right)$ and $2 q^{b-45} \mid(\pi+1)$. In either case, we see that neither $p$ nor $q$ can be the special prime.

In case (iii), when $q$ is a Fermat prime in $T$ (and $p$ is not), we compute

$$
2^{4^{9}}>10^{12} P^{2} N \geqslant 10^{12} \pi^{2} p^{a} q^{b} \frac{\sigma\left(p^{a} q^{b}\right)}{p^{k+3} q^{k+3}}>10^{12} q^{4 b-102} p^{2 a-12}>10^{12} p^{6 a-12} q^{-102} .
$$

When $p$ is a Fermat prime in $T$ (but $q$ is not) we similarly find

$$
2^{4^{9}}>10^{12} P^{2} N \geqslant 10^{12} \pi^{2} p^{a} q^{b} \frac{\sigma\left(p^{a} q^{b}\right)}{p^{k+3} q^{k+3}}>10^{12} p^{6 a-102} q^{-12}
$$

Finally, in case (i) we know $2 p^{a-45} q^{b-45} \mid(\pi+1)$. So we have

$$
2^{4^{9}}>10^{12} P^{2} N \geqslant 10^{12} \pi^{3} p^{a} q^{b} \frac{\sigma\left(p^{a} q^{b}\right)}{p^{k+3} q^{k+3}}>10^{12} p^{10 a-147} q^{-147} .
$$

The extra $\pi$ comes from $N$, since $\pi \nmid \sigma\left(p^{a} q^{b}\right)$ in this case. Solving for $a$ in the above inequalities yields the stated bounds.

The numbers in this proposition are not chosen to be the strongest possible, but rather to be convenient for the case $k=9$. If some of the hypotheses are strengthened then the proposition will work (with modified bounds) for larger $k$ and larger primes. The real strength of the proposition is in the fraction out front. We can use, with little loss in computational speed, the more uniform bound

$$
\frac{1}{\epsilon}\left(\frac{4^{k} \log (2)}{\log (p)}+C(k, q)\right),
$$


where $C(k, q)$ is some constant depending only on $k$ and $q$, and where $\epsilon$ is 4,6 , or 10 depending on the number of Fermat primes among $\{p, q\}$. Similar statements apply to the following proposition.

Proposition 2.5. Let $p$ and $q$ be odd, distinct primes less than 180 , and let $T=$ $\{3,5,17\}$. Let $N$ be an odd perfect number with $k \leq 9$ distinct prime divisors. Suppose $p^{a}\left\|N, q^{b}\right\| N$, and $10^{100}<p^{a}<q^{b}$ for some $a, b \in \mathbb{Z}_{>0}$.

(i) If $p, q \in T$, then

$$
b<\frac{1}{5}\left(\frac{4^{9} \log (2)-(5 a-147) \log (p)-12 \log (10)}{\log (q)}+147\right) .
$$

(ii) If $p \in T$ and $q \notin T$, then

$$
b<\frac{1}{2}\left(\frac{4^{9} \log (2)-(4 a-102) \log (p)-12 \log (10)}{\log (q)}+12\right) .
$$

(iii) If $p \notin T$ and $q \in T$, then

$$
b<\frac{1}{5}\left(\frac{4^{9} \log (2)-(a-12) \log (p)-12 \log (10)}{\log (q)}+135\right) .
$$

(iv) If $p, q \notin T$, then

$$
b<\frac{1}{2}\left(\frac{4^{9} \log (2)-(2 a-12) \log (p)-12 \log (10)}{\log (q)}+12\right) .
$$

Proof. One does an analysis as in the previous proposition. The only difficulty is deciding to use the lower bound $10^{12} P^{2} N \geq 10^{12} \pi^{2} p^{a} q^{b} \sigma\left(p^{a} q^{b}\right) /\left(p^{k+3} q^{k+3}\right)$ when $p \in T$ and $q$ is not, and to use $10^{12} P^{2} N \geq 10^{12} \pi^{3} p^{a} q^{b} \sigma\left(q^{b}\right) / p^{k+3}$ when $q \in T$ and $p$ is not. In case (i) we find

$$
2^{4^{9}}>10^{12} P^{2} N>10^{12} \pi^{3} p^{a} q^{b} \frac{\sigma\left(p^{a} q^{b}\right)}{p^{k+3} q^{k+3}}>10^{12} p^{5 a-147} q^{5 b-147} .
$$

Solving for $b$ gives the needed bound. The other cases are similar and are left to the reader.

Remark. One also has the inequality $b>a \frac{\log (p)}{\log (q)}$ in all cases, as $p^{a}<q^{b}$.

When searching through candidate odd perfect numbers $N$, one often can reduce to the case when $N$ is divisible by a prime power $p^{a} \| N$ with $a$ large. Using congruence conditions, when $a$ is large enough one can show that there exists a very big prime factor $Q_{1}$ of $N$. Historically, it was considerations such as this which led to the proofs in [15] and then [2,6] that odd perfect numbers must have seven, and then eight, distinct prime factors. In [13, the insight which improved the number of distinct prime factors to nine was that not only do congruence conditions yield a very large prime factor $Q_{1}$, but there must also be another large prime $Q_{1}^{\prime}>10^{11}$ (which, in practice, is not quite as large as $Q_{1}$ ) that divides $\sigma\left(p^{a}\right)$. If one can show the existence of a third large prime, further improvements can be made.

The propositions above are the key tool to finding a possible third large prime divisor. First, reduce to the case where we have two different prime powers $p^{a} \| N$ and $q^{b} \| N$ with $a, b$ large. The sizes of $a$ and $b$ are bounded above. We know that there should be a large prime divisor $Q_{1}^{\prime}$ of $\sigma\left(p^{a}\right)$ and a large prime divisor $Q_{2}^{\prime}$ for $\sigma\left(q^{b}\right)$. Our aim is to show that $Q_{1}^{\prime}$ and $Q_{2}^{\prime}$ are not equal. Thus, we compute $\operatorname{gcd}\left(\sigma\left(p^{a}\right), \sigma\left(q^{b}\right)\right)$ (for $a, b$ limited to the ranges given in the propositions above), 
and find that there are no common large primes. This computation was run in Mathematica, on a single core, over the course of a few months. We summarize the results of this computation as follows.

Theorem 2.6. Let $p$ and $q$ be odd, distinct primes, less than 105. Let $N$ be an odd perfect number with $k \leq 9$ distinct prime divisors. Suppose $p^{a}\left\|N, q^{b}\right\| N$, and $10^{100}<p^{a}<q^{b}$ for some $a, b \in \mathbb{Z}_{>0}$. Then the largest prime which divides both $\sigma\left(p^{a}\right)$ and $\sigma\left(q^{b}\right)$ is smaller than $10^{11}$.

The number $10^{11}$ was chosen to be compatible with the bounds developed in [13, and it was fortunate that it was sufficiently large to preclude the existence of counterexamples. While the existence of large common prime divisors is very scarce, there are still some close calls, such as 27866489501 dividing $\operatorname{gcd}\left(\sigma\left(p^{a}\right), \sigma\left(q^{b}\right)\right)$ with $p=59, a=2874, q=7$, and $b=15394$. Another close call occurred with the prime 17622719441 , for inputs $p=103, a=3598, q=61$, and $b=11833$. Even so, if we were to reduce $10^{11}$ to $10^{10}$ then we could still easily deal with those examples that arise. (The two just mentioned are the only two larger than $10^{10}$.)

The assumption in Theorem 2.6 that $N$ is an odd perfect number is used in two ways. First, we can use the bounds given in the previous propositions. Second, we may limit the exponents $a$ and $b$ even further (according to whether or not $p$ or $q$ can be the special prime). The code for these (and other) computations is available on the author's website.

\section{IMPROVEMENTS TO LEMMAS IN THE LITERATURE}

For the remainder of this paper we will assume that the reader is familiar with the paper [13. We will freely use the notations, definitions, and results given there. Some of the lemmas in that paper are streamlined to work for odd perfect numbers with $k \leq 8$ distinct prime factors. Some further computation is needed to allow the case $k=9$. Below is a full list of the changes we make to the methods employed in 13.

First, the precompiled table of factorizations of $\sigma\left(p^{a}\right)$, when $p$ and $a$ are small, requires extension. When $p<30$ the factorization of $\sigma\left(p^{a}\right)$ is entered into the table as long as $p^{a}<10^{200}$. If $30<p<105$, then we list factorizations when $p^{a}<10^{150}$. For the primes $105<p<10000$, we use the upper bound $p^{a}<10^{50}$. Finally, for primes $p>10^{4}$, factorizations are not put in a table, but rather we compute them as needed, and only compute them when $p^{a}<10^{30}$. The cutoffs $10^{200}, 10^{150}, 10^{50}$, and $10^{30}$ are used to decide when a prime power becomes infinite (in the sense of [13]). For the primes $p<30$, this bound is getting close to the best upper bound possible using current factoring algorithms.

Second, we improve Lemma 9 from that paper as follows:

Lemma 3.1. Let $p$ be an odd prime and let $q \in\{3,5,17\}$. If $q^{p-1} \equiv 1\left(\bmod p^{2}\right)$, then either $(q, p)=(3,11),(q, p)=(17,3)$, or $q^{o_{p}(q)}-1$ has a prime divisor greater than $10^{14}$.

Proof. If $p>10^{14}$, then $p$ is the needed prime divisor. If $p<10^{14}$, then there are only twelve pairs $(p, q)$ with $q^{p-1} \equiv 1\left(\bmod p^{2}\right)$, due to a computation reported in Mossinghoff's paper [11. Two cases are exceptional, and they appear in the statement of this lemma. For each of the other cases we compute all the prime divisors of $q^{o_{p}(q)}-1$ less than $10^{14}$, and see that the remaining cofactor is not 1 . 
Our third change is that Proposition 10 in [13] can be improved as follows.

Proposition 3.2. Let $N$ be an odd perfect number with $k, k_{1}$, and $k_{2}$ having their usual meanings. Suppose $q \in\{3,5,17\}$ is a known prime divisor of $N, q^{n} \| N$, $q \neq \pi$, and $\pi \nmid \sigma\left(q^{n}\right)$. Suppose $p_{1}, \ldots, p_{k_{1}-1}$ are the other known prime factors of $N$, besides $q$. For each $i=1,2, \ldots, k_{1}-1$ define

$$
\epsilon_{i}= \begin{cases}0 & \text { if } o_{p_{i}}^{\prime}(q)=0, \\ \max (s+t-1,1) & \text { if } o_{p_{i}}^{\prime}(q) \neq 0, \text { where } s=v_{p_{i}}\left(\sigma\left(q^{o_{p_{i}}}(q)-1\right)\right) \\ \text { and } t & \in \mathbb{Z}_{+} \text {is minimal so that } p_{i}^{t}>100 .\end{cases}
$$

Set $V=\prod_{i=1}^{k_{1}-1} p_{i}^{\epsilon_{i}}$. Suppose $\pi$ is among the $k_{2}$ unknown prime factors. Finally, assume that all unknown prime factors are greater than 100 .

If

$$
\left.\min \left(\sigma\left(q^{n}\right) / V\right), \sigma\left(q^{100} / V\right)\right)>1,
$$

then $k_{2}>1$. In that case, if

$$
\min \left(10^{14},\left(\frac{\sigma\left(q^{n}\right)}{V}\right)^{\frac{1}{k_{2}-1}},\left(\frac{\sigma\left(q^{100}\right)}{V}\right)^{\frac{1}{k_{2}-1}}\right)>1,
$$

then $\sigma\left(q^{n}\right)$ has a prime divisor among the unknown primes at least as big as this minimum.

Proof. There are two differences between this proposition and the original result. The easier change is that we replaced both $10^{11}$ and $10^{13}$ by $10^{14}$, which follows by citing Lemma 3.1 above. The other change is that instead of merely assuming $k_{2}>1$, we claim it is a consequence of the inequality $\left.\min \left(\sigma\left(q^{n}\right) / V\right), \sigma\left(q^{100} / V\right)\right)>$ 1. This new implication follows immediately from the ideas present in the original proof.

The fourth change is that after a short computation Lemma 12 in [13] now holds within the range $q<10^{4}$ and $p \geq \min \left(q^{n-2}, 10^{1000}\right)$. The exact statement of the improved result is the following.

Lemma 3.3. Let $q<10^{4}$ be an odd prime. If $p^{q-1} \equiv 1\left(\bmod q^{n}\right)$ for some $n \in \mathbb{Z}_{+}$ and some odd prime $p$, then $p \geq \min \left(q^{n-2}, 10^{1000}\right)$ except when

$$
(p, q)=(40663372766570611389846294355914421,7) .
$$

Note that the special case of this lemma causes no problems when $k=9$ since $o_{q}(p)=6$ and $\sigma\left(p^{5}\right)$ gives rise to twelve additional distinct prime factors of $N$.

Fifth, we improve Proposition 14 in 13 by replacing $10^{50}$ with $10^{1000}$. (This change merely involves a quick computation.) For completeness we include the full statement here.

Proposition 3.4. Let $N$ be an odd perfect number, and let $q<1000$ be a prime divisor of $N$ with $q^{n} \| N$. Suppose $b, k, k_{1}, k_{2}, \ell_{1}, \ell_{2}, k_{1}^{\prime}$, and $\ell_{1}^{\prime}$, have the same meanings as in [13. Proposition 7]. Suppose further that the exceptional case of the previous lemma doesn't hold. Let $T$ be the set of known primes with unknown component, different from $q$, and $\not \equiv 1(\bmod q)$. Let

$$
\begin{aligned}
\tau=n-b & -\sum_{p \in T, o_{q}^{\prime}(p) \neq 0}\left(v_{q}\left(p^{o_{q}(p)}-1\right)+\left\lfloor\frac{k_{1}^{\prime}+k_{2}}{\sigma_{0}\left(o_{q}(p)\right)}\right\rfloor\right) \\
& -\left(k_{1}^{\prime}-\ell_{1}^{\prime}+k_{2}\right)\left(k_{1}^{\prime}+k_{2}-1\right) .
\end{aligned}
$$


If $\tau>0$, then one of the unknown primes is not congruent to $1(\bmod q)$. Further, in this case, one of the unknown primes is at least as large as $\min \left(q^{\tau^{\prime}-2}, 10^{1000}\right)$ where

$$
\begin{aligned}
\tau^{\prime}= & \min _{1 \leqslant m \leqslant k_{2}}\left\lceil\left( n-b-\sum_{p \in T, o_{q}^{\prime}(p) \neq 0}\left(v_{q}\left(p^{o_{q}(p)}-1\right)+\left\lfloor\frac{k_{1}^{\prime}+k_{2}-m}{\sigma_{0}\left(o_{q}(p)\right)}\right\rfloor\right)\right.\right. \\
& \left.\left.-\left(k_{1}^{\prime}-\ell_{1}^{\prime}+k_{2}-m\right)\left(k_{1}^{\prime}+k_{2}-m-1\right)-m\left\lfloor\frac{k_{1}^{\prime}+k_{2}-m}{2}\right\rfloor\right) / m\right] .
\end{aligned}
$$

Sixth, we can replace Lemma 15 in 13 , with the following version (with the same proof, mutatis mutandis).

Lemma 3.5. Let $q$ be a prime with $7 \leq q<1000$. Suppose $a^{q-1} \equiv 1\left(\bmod q^{n}\right)$ for some $n \in \mathbb{Z}_{>0}$ and some positive integer $a$ with $(q-1) \mid a$. Then $a \geq \min \left(q^{n-4}, 10^{1000}\right)$.

At this point, all of the changes we have made have consisted mainly of minor improvements in a few constants. These are achieved by performing computations which take a day or two on a single core. The seventh change is a significant improvement to Lemma 16 in 13 , which takes a great deal of machine power. I wish to thank William Lipp for helping distribute the needed factoring over the internet, and also thank those who helped in that effort. The improved lemma reads as follows:

Lemma 3.6. Let $p$ and $q$ be primes with $10^{2}<p<10^{11}$ and $7 \leq q<180$. If $q^{p-1} \equiv 1\left(\bmod p^{2}\right)$, then $\sigma\left(q^{o_{p}(q)-1}\right)$ is divisible by two primes greater than $10^{11}$.

Proof. The paper [10] lists all 61 pairs $(p, q)$ satisfying the conditions of the lemma. It should be mentioned that Richard Fischer has recently improved the range of these computations, and the interested reader is directed to his website: http:// www. fermatquotient. com/FermatQuotienten/

For 56 of those pairs, we have two explicit prime factors of $A=\sigma\left(q^{o_{p}(q)-1}\right)$, each greater than $10^{11}$. For another 3 pairs, we have one such prime factor $P$ for which $P^{2} \nmid A$. Further, after computing all prime factors of $A / P$ less than $10^{11}$ we find that the cofactor is not 1 , so there must exist some other prime factor greater than $10^{11}$. The explicit factors are listed on Lipp's website http://home.earthlink. net/ oddperfect/FermatQuotients.html and are also available on the author's personal website.

The remaining two pairs are $(p, q)=(1025273,41)$ and $(q, p)=(122327,157)$. In the first case $o_{p}(q)=2^{3} \cdot 128159$. Exhaustively removing all of the prime factors of $41^{128159}-1$ less than $10^{11}$, we find a non-trivial cofactor. Similarly, $41^{128159}+1$ also has a prime divisor larger than $10^{11}$. Both of these primes divide $A$. This deals with the first case. The second case is dealt with similarly, as $o_{p}(q)=2 \cdot 1973$.

It should be mentioned that this lemma can be improved further. Indeed, if $p$ and $q$ are odd primes in the (larger) ranges $q<1000$ and $p<10^{11}$, still satisfying $q^{p-1} \equiv 1\left(\bmod p^{2}\right)$, then once again all possible pairs $(p, q)$ are known. In many cases we can again find two explicit prime factors greater than $10^{11}$, or find one explicit factor and prove the existence of a second such prime. However, there are 29 cases, such as $(p, q)=(101,181)$, for which one, and only one, prime factor larger than $10^{11}$ exists. There are also 166 cases, such as $(p, q)=(3,19)$, for which no large prime factors exist. (In those cases usually $p$ is very small, which is why in the 
lemma above we restricted to the range $p>100$.) Finally, there are 4 cases, such as $(p, q)=(3443059,281)$, where it is likely that there exist two large prime factors, but we didn't explicitly prove it because there was no need. (However, one can use a variant of the upper bound $P^{2} N<2^{4^{k}}$ to easily deal with these remaining cases, at least when $k=9$.)

The eighth change is that the statement of Proposition 17 in 13 can be modified a great deal, with the only significant change to the proof that we now cite Lemma 3.6. Here is the precise improved result.

Proposition 3.7. Let $N$ be an odd perfect number and let $7 \leq q<180$ be a known prime divisor of $N$, with $q^{n} \| N$. Let $\tau, \tau^{\prime}$ be as in Proposition 3.4, suppose all the hypotheses of that proposition are met, and let $p$ be the guaranteed unknown prime. Let $p_{1}, \ldots, p_{k_{1}-1}$ be the known primes different from $q$. Let $\epsilon_{i}$ be defined as before, and put $V=\prod_{i=1}^{k_{1}-1} p_{i}^{\epsilon_{i}}$. Finally, assume that all unknown prime factors are greater than 100.

$$
\begin{aligned}
& \text { If } \left.\min \left(\sigma\left(q^{\tau^{\prime}-4}\right) / V\right), \sigma\left(q^{96} / V\right)\right)>1 \text {, then } k_{2}>1 \text {. In that case, if } \\
& \qquad \min \left(10^{11},\left(\frac{q^{96}}{(q-1) V}\right)^{\frac{1}{k_{2}-1}},\left(\frac{q^{\tau^{\prime}-4}}{(q-1) V}\right)^{\frac{1}{k_{2}-1}}\right)>1,
\end{aligned}
$$

then $\sigma\left(q^{n}\right)$ has a prime divisor, different from $p$, among the unknown primes, at least as big as the above minimum.

Proof. The range of the prime $q$ has been improved from $q \in\{7,11,13\}$ to the range $7 \leq q<180$, which we can do by Lemma 3.6. However, the quantity $q^{\tau^{\prime}-2}$ from the original result has been replaced by the slightly worse $q^{\tau^{\prime}-4}$ (essentially, to deal with $q=7$ ). The constant $10^{50}$ has been superseded by $q^{96}$ (noting $q^{96}<10^{1000}$ ). Similar to Proposition 3.2. the assumption " $k_{2}>1$ " has been replaced with the statement "if $\left.\min \left(\sigma\left(q^{\tau^{\prime}-4}\right) / V\right), \sigma\left(q^{96} / V\right)\right)>1$, then $k_{2}>1$ " which is proved in an analogous manner.

Note that if $\left.\min \left(\sigma\left(q^{\tau^{\prime}-4}\right) / V\right), \sigma\left(q^{96} / V\right)\right)>1$ holds but $k_{2}=1$, then we reach a contradiction. So we add this contradiction to the list found in [13, Section 7]. (A similar statement holds for Proposition 3.2.)

The final change in our implementation of the algorithm in 13 is that when applying Lemma 20 to find upper bounds on the next unknown prime, we use Theorem 2.6 above to contribute more large primes. However, Theorem 2.6 does not automatically prove the existence of a third large prime. In practice, we have one prime $Q_{1}$ coming from congruence conditions relative to $p$, and another prime $Q_{1}^{\prime}>10^{11}$ dividing $\sigma\left(p^{a}\right)$ with $Q_{1}^{\prime} \neq Q_{1}$. Similarly, we have one prime $Q_{2}$ from congruence conditions relative to $q$, and another prime $Q_{2}^{\prime}>10^{11}$ dividing $\sigma\left(q^{b}\right)$ with $Q_{2}^{\prime} \neq Q_{2}$. Theorem 2.6 asserts $Q_{1}^{\prime} \neq Q_{2}^{\prime}$, but it could still be the case that $Q_{2}=Q_{1}^{\prime}$ and $Q_{1}=Q_{2}^{\prime}$.

When computing the bounds coming from Lemma 20 in 13 , we are thus led to consider two situations, which we describe now. Suppose our algorithm has reached a point where we have a list $\left\{q_{1}^{a_{1}}, q_{2}^{a_{2}}, \ldots, q_{n}^{a_{n}}\right\}$ of infinite prime powers in a suspected odd perfect number. Let $Q_{i}$ be the large prime coming from $q_{i}$ from the congruence conditions in [13, Proposition 7] or Proposition 3.4 (according to whether $q_{i} \in\{3,5,17\}$, or not). Similarly, let $Q_{i}^{\prime}$ be the second large prime coming 
from $q_{i}$, using Proposition 3.2 or 3.7. We drop from our list any $q_{i}$ for which $Q_{i}^{\prime}<10^{11}$, so that Theorem 2.6 will apply.

There are now two main cases. We apply Lemma 20 in both cases, and then use the lesser of the two bounds achieved. One option is that $Q_{1}=Q_{2}=\cdots=Q_{n}$. In this case, as each $Q_{i}^{\prime} \neq Q_{i}=Q_{1}$ and $Q_{i}^{\prime} \neq Q_{j}^{\prime}$ by Theorem 2.6, we can apply Theorem 20 with the bounds $\left\{P_{1}=Q_{1}, P_{2}=10^{11}, P_{3}=10^{11}, \ldots, P_{n+1}=10^{11}\right\}$. The second option is that the $Q_{i}$ are not all equal. Let $Q_{1}$ be the largest element in $\left\{Q_{1}, Q_{2}, \ldots, Q_{n}\right\}$, and let $Q_{n}$ be the smallest. The worst possible case would be that among the distinct primes $\left\{Q_{1}^{\prime}, Q_{2}^{\prime}, \ldots, Q_{n}^{\prime}\right\}$ the two largest primes are equal to $Q_{1}$ and $Q_{n}$, and the rest are close to $10^{11}$. Thus, we apply Theorem 20 with the list of bounds $\left\{P_{1}=Q_{1}, P_{2}=Q_{n}, P_{3}=10^{11}, \ldots, P_{n}=10^{11}\right\}$.

With all of these changes in place, we rerun the algorithm described in 13 . The computation takes just over one day on a single core, covering a little over thirty million cases, and we achieve:

Theorem 3.8. There are no odd perfect numbers with less than 10 distinct prime factors.

There are a few problem cases requiring special treatment, which we describe now. These cases also illustrate some of the benefits and limitations in the changes we made above.

Initially, the plan had been to make the primes $p=101,103$ become infinite when $p^{a}>10^{50}$. However, with this choice the prime power $103^{\infty}$ never satisfies the bounds in Proposition 3.4. This left cases such as $3^{\infty} 5^{\infty} 19^{\infty} 103^{\infty} 1399^{\infty} p^{\infty}$ (where $13689227 \leq p \leq 13691033$ ). Here we needed another large prime, since the bounds on the next unknown prime were already bigger than $10^{11}$. This problem was solved by expanding the factorization table for $p=101,103$ up to the level $p^{a} \leq 10^{150}$.

This still leaves five problem cases:

- $3^{4} 11^{\infty} 5^{1} 73^{\infty} 2633^{\infty} 1157609^{\infty}$.

- $3^{4} 11^{\infty} 5^{1} 73^{\infty} 2633^{\infty} 1157621^{\infty}$.

- $3^{4} 11^{\infty} 5^{1} 73^{\infty} 2633^{\infty} 1157627^{\infty}$.

- $3^{4} 11^{\infty} 5^{1} 97^{\infty} 263^{\infty} 575513^{\infty}$.

- $3^{4} 11^{\infty} 5^{1} 103^{\infty} 227^{\infty} 349667^{\infty}$.

We will only discuss the first case, as the other four are dealt with similarly. In that case, the next unknown prime $p$ is given inside an interval $249075961044<$ $p<498151922091$. This interval contains more than nine billion primes, which is too many to check one at a time. The reason for the extremely large interval is that the upper (and lower) bound on the interval is larger than $10^{11}$, and thus falls outside the scope of the bounds in Proposition 3.7. When this project was begun, the number $10^{11}$ was the bound initially chosen when proving Lemma 3.6, and thus subsequently used in many other lemmas and propositions.

There are at least two ways to deal with this case. First, one could redo the computation of Theorem 2.6 to include the pairs

$$
(p, q) \in\{(11,2633),(73,2633),(2633,11),(2633,73)\},
$$

and then modify Lemma 3.6. and all subsequent results, to include the new prime 2633. A second option is to again modify Lemma 3.6, and all subsequent results, but this time just for the primes 11 and 73 , by replacing the bound $10^{11}$ with $10^{12}$. The 
second option is straightforward and easy, except for extending the computations in 10. Fortunately, as we already mentioned, such an extension has already been performed by Richard Fischer (up to the even better bound $8.2 \times 10^{12}$ ). This finishes the first special cases. The other four are dealt with similarly.

\section{CAN We Prove $k>10$ ?}

The simple answer to the question in the title of this section is no. We will give an illustrative example, demonstrating one of the many difficulties one would face in trying to extend the computations further. Consider the case $3^{2} 13^{2} 61^{\infty} 5^{\infty} 163^{\infty}$ $121309^{\infty} 4328041097^{\infty}$. The next unknown prime lies in the interval $1.2 \times 10^{19}<$ $p<2.6 \times 10^{19}$. It would be quite difficult (if not impossible with current computing resources) to improve Lemma 3.1 for the prime $q=5$ to achieve a bound $2.6 \times 10^{19}$, much less also improve Lemma 3.6 similarly to $2.6 \times 10^{19}$ for the primes $q=61,163$. We would need to do this for at least two of these three primes, to use the previous methods to decrease the size of the interval.

A related problem is whether these techniques can be used to show that when $3 \nmid N$, the bound $k \geq 12$ can be improved. This may be possible, but it would require an improvement to Theorem 2.6 from $k \leq 9$ to $k \leq 12$, which roughly translates into a sixty-four fold increase in computation time. As the original calculation to prove Theorem 2.6 took several months, this improvement would only be feasible using a massive effort distributed over many computers.

\section{ACKNOWLEDGEMENTS}

The author thanks William Lipp for many useful comments on manuscripts, and for spearheading the factorization efforts which led to Lemma 3.6. The author also thanks Samuel Dittmer and Mitsuo Kobayashi for conversations which led to some improvements in Lemma 1.2, and Yong-Gao Chen for providing a preprint version of the paper [3]. Many of the initial computations used in this paper were performed while the author was partially supported by the University of Iowa Department of Mathematics NSF VIGRE grant DMS-0602242. This work was partially supported by a grant from the Simons Foundation (\#315828 to Pace Nielsen). Finally, the author thanks the anonymous referees for comments and suggestions which improved the paper immensely.

\section{REFERENCES}

[1] R. P. Brent, G. L. Cohen, and H. J. J. te Riele, Improved techniques for lower bounds for odd perfect numbers, Math. Comp. 57 (1991), no. 196, 857-868, DOI 10.2307/2938723. MR.1094940 (92c:11004)

[2] J. E. Z. Chein, An Odd Perfect Number has at Least 8 Prime Factors, ProQuest LLC, Ann Arbor, MI, 1979. Thesis (Ph.D.)-The Pennsylvania State University. MR2630408

[3] Y.-G. Chen, Improved upper bounds for odd multiperfect numbers, Bull. Aust. Math. Soc. 89 (2014), no. 3, 353-359, DOI 10.1017/S0004972713000488. MR3254745

[4] R. J. Cook, Bounds for odd perfect numbers, Number theory (Ottawa, ON, 1996), CRM Proc. Lecture Notes, vol. 19, Amer. Math. Soc., Providence, RI, 1999, pp. 67-71. MR.1684591 (2000d:11010)

[5] S. J. Dittmer, Spoof odd perfect numbers, Math. Comp. 83 (2014), no. 289, 2575-2582, DOI 10.1090/S0025-5718-2013-02793-7. MR3223347

[6] P. Hagis Jr., Outline of a proof that every odd perfect number has at least eight prime factors, Math. Comp. 35 (1980), no. 151, 1027-1032, DOI 10.2307/2006211. MR572873 (81k:10004) 
[7] D. R. Heath-Brown, Odd perfect numbers, Math. Proc. Cambridge Philos. Soc. 115 (1994), no. 2, 191-196, DOI 10.1017/S0305004100072030. MR1277055 (96b:11130)

[8] D. E. Iannucci, The second largest prime divisor of an odd perfect number exceeds ten thousand, Math. Comp. 68 (1999), no. 228, 1749-1760, DOI 10.1090/S0025-5718-99-01126-6. MR.1651761(2000i:11200)

[9] D. E. Iannucci, The third largest prime divisor of an odd perfect number exceeds one hundred, Math. Comp. 69 (2000), no. 230, 867-879, DOI 10.1090/S0025-5718-99-01127-8. MR 1651762 (2000i:11201)

[10] W. Keller and J. Richstein, Solutions of the congruence $a^{p-1} \equiv 1\left(\bmod p^{r}\right)$, Math. Comp. 74 (2005), no. 250, 927-936 (electronic), DOI 10.1090/S0025-5718-04-01666-7. MR2114655 (2005i:11004)

[11] M. J. Mossinghoff, Wieferich pairs and Barker sequences, Des. Codes Cryptogr. 53 (2009), no. 3, 149-163, DOI 10.1007/s10623-009-9301-3. MR2545689 (2011c:11039)

[12] P. P. Nielsen, An upper bound for odd perfect numbers, Integers 3 (2003), A14, 9. MR2036480 (2004k:11009)

[13] P. P. Nielsen, Odd perfect numbers have at least nine distinct prime factors, Math. Comp. 76 (2007), no. 260, 2109-2126, DOI 10.1090/S0025-5718-07-01990-4. MR.2336286 (2008g:11153)

[14] P. Ochem and M. Rao, Odd perfect numbers are greater than $10^{1500}$, Math. Comp. 81 (2012), no. 279, 1869-1877, DOI 10.1090/S0025-5718-2012-02563-4. MR2904606

[15] C. Pomerance, Odd perfect numbers are divisible by at least seven distinct primes, Acta Arith. 25 (1973/74), 265-300. MR0340169 (49 \#4925)

[16] J. Voight, On the nonexistence of odd perfect numbers, MASS selecta, Amer. Math. Soc., Providence, RI, 2003, pp. 293-300. MR2027187 (2004j:11006)

Department of Mathematics, Brigham Young University, Provo, Utah 84602

E-mail address: pace@math.byu.edu 